\title{
Field assessment of the efficacy of M.B., LIBDV and Winterfield 2512 strain vaccines against infectious bursal disease in chickens
}

\author{
Ho M. Nguyen ${ }^{1}$, Anh T. Quach ${ }^{1 *}$, Anh T. T. Le ${ }^{2}, \&$ Hien T. Le \\ ${ }^{1}$ Faculty of Animal Science and Veterinary Medicine, Nong Lam University, Ho Chi Minh City, Vietnam \\ ${ }^{2}$ Vetstar, Ho Chi Minh City, Vietnam
}

\author{
ARTICLE INFO \\ Research Paper \\ Received: October 12, 2018 \\ Revised: November 09,2018 \\ Accepted: November 28, 2018
}

\section{* Corresponding author}

Quach Tuyet Anh Email: anh.quachtuyet@hcmuaf.edu.vn

\begin{abstract}
Live virus vaccines are very important parts of the prevention of Infectious Bursal Disease (IBD) in chickens. However, the successful IBD vaccination depends on IBD field pressure, vaccination technique, the immune status of the chicken, and especially IBDV strains used in the vaccines which are able to break through a higher level of maternal-derived antibodies (MDA). The objective of this field study was to compare the efficacy of a new vaccine based on M.B. strain to other commercial vaccines (LIBDV and winterfiled 2512) in terms of speed of antibody immune response and interference to Newcastle Disease (ND) vaccination. Six houses of broilers, each with 15,000 to 16,000 chickens, were divided into two groups: (1) vaccinated with M.B. strain (group A) and (2) vaccinated with LIBDV or 2512 strains (group B). Blood samples were collected prior to the $1^{\text {st }}$ IBD vaccination, and at 21, 28 and 35 days of age for IBD and ND antibodies. Comparison of lesion scores and uniformity of the bursa of Fabricius (BF) at 28 and 35 days of age was carried out. Results showed that both groups had good immune responses, but group A showed significantly higher IBD antibody titers at 28 and 35 days of age. Antibody titers for ND and histopathological lesion scores of the BF were not significantly different between the 2 groups. The BF in group A was more uniform and had fewer lesions when compared with that in group B. In conclusion, the IBD vaccine with an M.B. strain can provide better immunological efficacy than LIBDV and 2512 strains.
\end{abstract}

Cited as: Nguyen, H. M., Quach, A. T., Le, A. T. T., \& Le, H. T. (2018). Field assessment of the efficacy of M.B., LIBDV and Winterfield 2512 strain vaccines against infectious bursal disease in chickens. The Journal of Agriculture and Development 17(6), 15-23.

\section{Introduction}

For many years, Infectious Bursal Disease (IBD) has been a serious problem threatening the poultry industry (Berg, 2000; Alkie \& Rautenschlein, 2016). In March 2018, a report from ILDEX Vietnam showed that $12 / 64$ provinces (18.75\%) in Viet Nam in 2017 had IBD disease outbreaks. Live vaccines have been proved to be a powerful tool in controlling Gumboro disease (Van den Berg et al., 2000; Eterradossi \& Saif, 2008; Muller et al., 2012). Currently, There are
3 types of live IBD vaccines available for young chickens including live attenuated IBD vaccines, immune complex vaccines, and recombinant vaccines (Gardin et al., 2011; Muller et al., 2012). However, IBD live vaccine is still a good solution for high vvIBD challenge areas (Berg \& Meulemans, 1991). This type of vaccine protects chickens as the vaccine viruses replicate at the bursa of fabricius and induce a strong immune reaction leading to high antibody titers, and the shedding of vaccine virus to the environment helps reduce the field virus pressure at farms (Gomes et al., 
2015). In addition, live IBD virus vaccine is very important for primary vaccination of the pullet. When stimulating memory cells, it acts as a good primer to inactivated IBD vaccination (Gardin et al., 2011). It is necessary to have high IBD antibody titers in the breeders that then pass to the offspring protecting them in the first 23 weeks of life (Eterradossi \& Saif, 2008; Fantay et al., 2015). Before the maternal-derived antibodies (MDA) drop too low, vaccinating broilers is really the key to the continuation of the protection (Fantay et al., 2015; Jackwood, 2017). For that reason, live IBD vaccines should have the ability to break through high levels of MDA to provide as early protection as possible without being neutralized by the MDA (Fantay et al., 2015). The new IBD vaccine strain in Vietnam market is M.B., Israeli strain, isolated in 1989 by Abic scientists Drs Barbakov and Gutter. The name of M.B. was named by these scientists. It got the United States patent on Sep. 8, 1998 with the patent number 5804195 . The M.B. strain belongs to genetic group 6 (Lazarus et al., 2008) and is able to break through MDA levels in broilers of 800 IDEXX ELISA while the normal intermediate IBD vaccines achieve this at a titer of 125 IDEXX ELISA and intermediate plus IBD vaccines at 500 IDEXX ELISA (De Wit, 2001).

The objective of this study was to evaluate the efficacy of the IBD M.B. vaccine strain and compare it with other commercial vaccines (LIBDV strain and 2512 strains) in commercial broiler chickens.

\section{Materials and Methods}

\subsection{Experimental design}

Six flocks (A1, A2, B1, B2, B3, and B4) (Figure 1) were kept in environmentally controlled broiler houses on 2 different commercially operated farms in Xuan Loc, Dong Nai. The flocks (A1, A2, B1, B2) were raised in farm 2 which was about 4 kilometers away from farm 1 (B3 and B4 flocks). There were 15,000 - 16,000 broiler chickens in each house. All chickens in farm 1 and 2 across the groups were subjected to the same management procedures and housing conditions.

A total of 95,000 day-old-chicks (Ross 308) used in this study were bought from the same breeding company, and hence they were assumed inherited the same MDA. This assumption was confirmed by testing the amount of MDA in chicks at 2 days old.

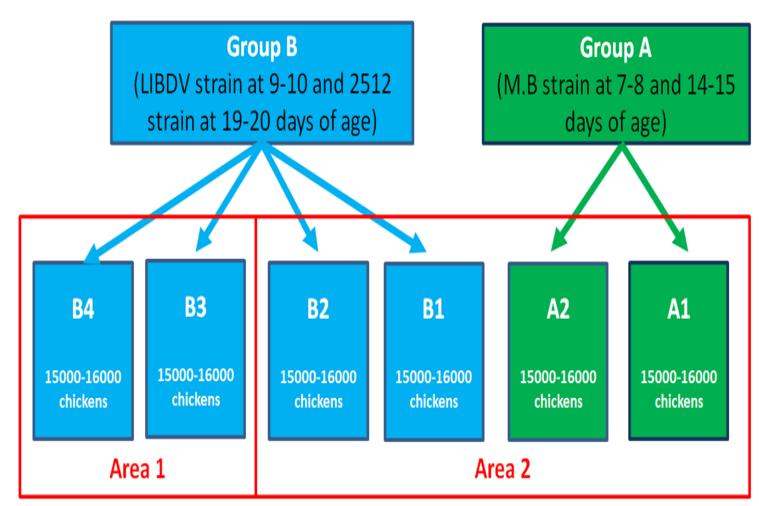

Figure 1. Design of experiments.

\subsection{Vaccination schedule}

There were two vaccination schedules which were different based on the Gumboro vaccination program (Table 1). All other vaccinations were exactly the same in both schedules. Schedule B was kept exactly as the vaccination program which was used in this farm for long time. It was considered as the standard vaccination program which was suitable for the condition and epidemiology of this farm. Schedule A was new set up based on the MDA levels at 2 days old. M.B. strain is able to break through MDA levels of 800 IDEXX ELISA, therefore, in schedule $\mathrm{A}$, the $1^{\text {st }}$ vaccination can be vaccinated as early as 7-8 days old. While LIBDV strain is able to break through MDA levels of less than 500 IDEXX ELISA, therefore, in schedule $B$, the $1^{\text {st }}$ vaccination should be later (9-10 days old). The $2^{\text {nd }}$ vaccination was 7-10 days after the $1^{\text {st }}$ vaccination (Table 1 ).

\subsection{Serology}

Blood samples from twenty chickens per house were randomly collected immediately prior to the $1^{\text {st }}$ IBD vaccination and subsequently at 21,28 and 35 days of age for determination of IBD and ND antibodies. A commercial enzyme-linked immunosorbent assay kit (IDEXX, Maine, USA, Cat. number 99-09260) was used as described by the manufacturer for the detection of antibodies to IBD in chicken serum. The ND antibody titers were measured by the haemagglutination inhibition method according to Allan \& Gough (1974) 
Table 1. Vaccination schedule

\begin{tabular}{|c|c|c|c|c|}
\hline \multirow{2}{*}{\multicolumn{2}{|c|}{$\begin{array}{l}\text { Schedule A } \\
\text { M.B. strain }\end{array}$}} & \multicolumn{2}{|c|}{ Schedule B } & \multirow{3}{*}{ Admission route } \\
\hline & & \multicolumn{2}{|c|}{ LIBDV and 2512 trains } & \\
\hline Day old & Vaccine $^{1}$ & Day old & Vaccine $^{1}$ & \\
\hline & $\mathrm{ND}-\mathrm{IB}$ & & $\mathrm{ND}-\mathrm{IB}$ & Spray \\
\hline 1 & ND kill & 1 & ND kill & $\mathrm{SC}$ \\
\hline $7-8$ & $\mathrm{MB}\left(1^{\mathrm{st}}\right)$ & $9-10$ & $\operatorname{LIBDV}\left(1^{\text {st }}\right)$ & DW \\
\hline 13 & $\mathrm{ND}-\mathrm{IB}$ & $13-14$ & ND - IB & DW \\
\hline $14-15$ & $\operatorname{MB}\left(2^{\text {nd }}\right)$ & $19-20$ & $2512\left(2^{\text {nd }}\right)$ & DW \\
\hline $27-28$ & $\mathrm{ND}-\mathrm{IB}$ & $27-28$ & ND - IB & DW \\
\hline
\end{tabular}

${ }^{1}$ SC: Subcutaneous; DW: Drinking water; ND: Newcastle disease; IB: Infectious bronchitis disease.

\subsection{Bursa of Fabricius analysis}

Five chickens per house unit were sacrificed at 28 and 35 days of age. Bursa index (BI) was calculated as bursa weight / body weight $\times 100$ (Sellaoui et al., 2012). Bursa size vs. spleen size of the same chicken and lesions of BF were observed and compared among chickens of the same housing unit.

The BF samples were fixed in $10 \%$ formalin and stained with hematoxylin and eosin described by Fischer et al. (2008). Lesions were observed microscopically and were evaluated based on the scoring system from 0 to 5 described by Muskett et al. (1979).

\subsection{Statistical analysis}

Collected data were managed and simple calculation were performed in MS Excel 2010. Random effect models were used to detect any influence of factors: age, or vaccine on antibody levels or Bursa indices. These models were built in Stata 11 with farm factors as random variables.

\section{Results}

\subsection{Maternally derived IBD antibodies}

Right before the $1^{\text {st }}$ IBD vaccination was applied, 20 serum samples from birds that were randomly selected from each house were measured to determine their maternal ELISA antibody titers. The titers ranged from 100 to 2614 in group A1, from 271 to 2534 in group A2, from 175 to 2320 in group B1 and from 264 to 2673 in group B2. Although the day of the $1^{\text {st }}$ IBD vaccination was different among houses (Table 1), the maternal antibody levels at the day of the $1^{\text {st }}$ IBD vaccina- tion in all houses in farm 2 were not significantly different $(P>0.05)$ (Table 2$)$.

\subsection{Induction of circulating IBD antibodies post-vaccination}

At 28 days of age, circulating ELISA IBD antibodies ranged from 227 to 3190 in group A1 (mean titer: 1854, CV: 46\%), from 140 to 4648 in group A2 (mean titer: 2431, CV: 47\%), from 46 to 3182 in group B1 (mean titer 789, CV: 118\%), from 0 to 4188 in group B2 (mean titer: 500, CV: $194 \%$ ), from 0 to 2315 in group B3 (mean titer: 885, CV:106\%) and from 972 to 2638 in group B4 (mean titer: 1787, CV: $34 \%$ ). At 28 days of age, the induction of an active immune response in group A were significantly higher than in group B $(P<0.001)$ (Table 2$)$. In addition, the antibody titers of the majority of samples in group A were above 1500 . On the contrary, the antibody titers of the majority of samples in group B were below 1500 (Figure 2). The CV in group A was also much lower than that in group B (Table 2 ), which showed that group A was much more uniform than group B.

At 35 days of age, circulating ELISA IBD antibodies ranged from 682 to 4700 in group A1 (mean titer: 2741, CV: 36\%), from 2014 to 3823 in group A2 (mean titer: 2851, CV: 20\%), from 802 to 5890 in group B1 (mean titer: 2066, CV: $54 \%$ ), from 33 to 5721 in group B2 (mean titer: 3127, CV: $51 \%$ ), from 1346 to 4999 in group B3 (mean titer: 3174, CV: 32\%) and from 1027 to 3666 in group B4 (mean titer: 2182, CV: $33 \%$ ). The induction of an active immune response in group A was significantly higher than in group B $(P<0.01)$ (Table 2$)$. Again, the $\mathrm{CV}$ in group $\mathrm{A}$ was still lower than that in group B (Table 2). 
Table 2. Induction of circulating IBD antibodies

\begin{tabular}{lccccccc}
\hline & \multicolumn{2}{c}{ Group A } & \multicolumn{5}{c}{ Group B } \\
& \multicolumn{2}{c}{ M.B. strain } & \multicolumn{5}{c}{ LIBDV + 2512 strains } \\
\cline { 2 - 8 } & Mean titer & CV $(\%)$ & N & Mean titer & CV $(\%)$ & N & $P$ \\
\hline IBD - before vac & 1083.62 & 59.06 & 40 & 1158.2 & 49.37 & 40 & 0.583 \\
IBD - 28 days old & 2142.25 & 48.66 & 40 & 991.57 & 99.47 & 80 & 0.000 \\
IBD - 35 days old & 2795.77 & 28.51 & 40 & 2637.13 & 47.25 & 80 & 0.010 \\
\hline
\end{tabular}

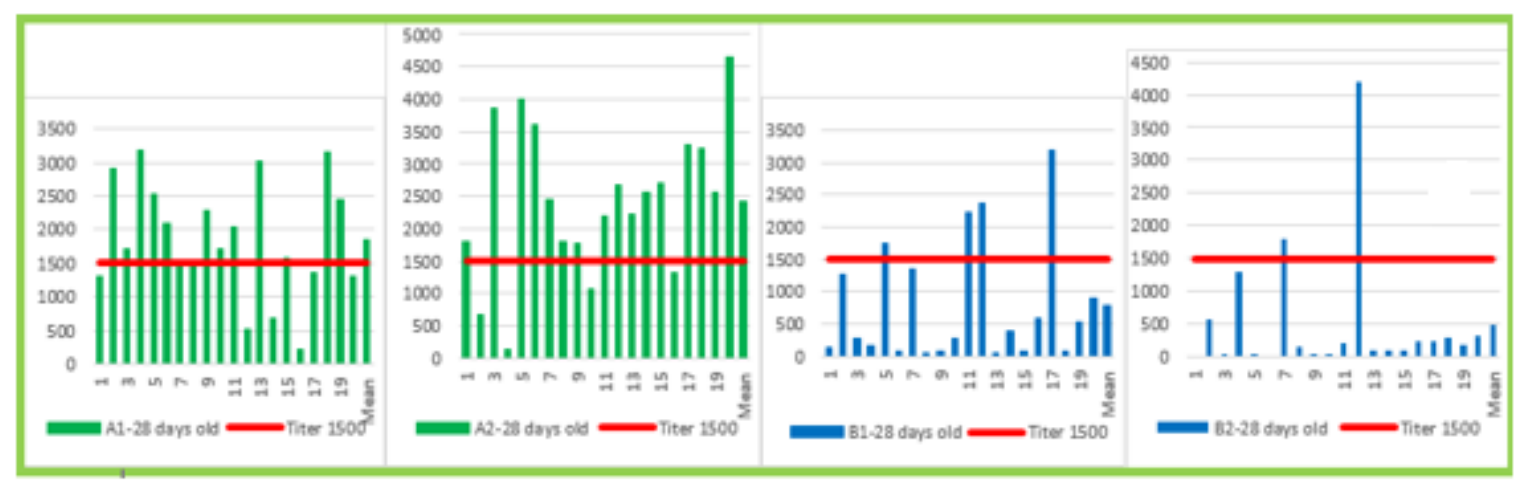

Figure 2. The serum IBD antibodies of chickens at 28 days old in farm 2 .

Table 3. Induction of circulating ND antibodies

\begin{tabular}{cccccccc}
\hline & \multicolumn{2}{c}{ Group A } & \multicolumn{4}{c}{ Group B } \\
& \multicolumn{2}{c}{ M.B. strain } & \multicolumn{4}{c}{ LIBDV + 2512 strains } \\
\cline { 2 - 8 } & Mean titer & CV $(\%)$ & N & Mean titer & CV $(\%)$ & N & $P$ \\
\hline ND - 21 days old & 3.30 & 39.00 & 20 & 1.65 & 49.00 & 20 & 0.000 \\
ND - 28 days old & 3.35 & 49.00 & 40 & 3.45 & 57.39 & 80 & 0.777 \\
ND - 35 days old & 5.77 & 32.68 & 40 & 4.17 & 49.80 & 80 & 0.255 \\
\hline
\end{tabular}

Table 4. Bursa index

\begin{tabular}{cccccccc}
\hline \multicolumn{3}{c}{ Group A } & \multicolumn{5}{c}{ Group B } \\
\hline & BI & CV (\%) & N & BI & CV (\%) & N & $P$ \\
\hline 28 & 0.56 & 26.5 & 10 & 1.02 & 57.7 & 20 & 0.015 \\
35 & 0.49 & 22.4 & 10 & 0.60 & 37.3 & 20 & 0.131 \\
\hline
\end{tabular}

\subsection{Induction of circulating ND antibodies} post-vaccination

At 21 days of age, the induction of an active ND immune response in group A were significantly higher than that in group $\mathrm{B}(P<0.001)$. However, at 28 and 35 days of age, the ND antibody titers of both groups were not significantly different $(P>0.05)$ (Table 3$)$.

\subsection{BI and the uniformity and lesions of BF}

At 28 days of age, the BI of group A was significantly smaller than group B $(P<0.05)$. How- ever, at 35 days of age, the BI of both groups were not significantly different $(P>0.05)$. Group A at both 28 and 35 days of age had fewer lesions (hemorrhages) than group B (Table 4). All data including CV (Table 4), comparing the size of bursa and spleen of the same chicken (Table 5) showed that the size of the bursa of group A was much more uniformity than group B's.

\subsection{Histopathology studies}

The score lesions of both groups at 28 and 35 days of age were not significantly different $(P>$ 0.05) (Table 6). 
Table 5. Detecting bursa lesions and comparing the size of bursa and spleen of the same chicken

\begin{tabular}{lcccc}
\hline & \multicolumn{2}{c}{28 days old } & \multicolumn{2}{c}{35 days old } \\
\cline { 2 - 5 } & $\begin{array}{c}\text { Group A } \\
(\mathrm{N}=10)\end{array}$ & $\begin{array}{c}\text { Group B } \\
(\mathrm{N}=20)\end{array}$ & $\begin{array}{c}\text { Group A } \\
(\mathrm{N}=10)\end{array}$ & $\begin{array}{c}\text { Group B } \\
(\mathrm{N}=20)\end{array}$ \\
\hline Hemorrhage inside (\%) & 10 & 20 & 0 & 15 \\
Bigger than spleen (\%) & 0 & 15 & 0 & 5 \\
Smaller or same size with spleen's $=$ normal & 100 & 75 & 100 & 95 \\
size (\%) & 0 & 10 & 0 & 0 \\
Bursa atrophy* (\%) & & &
\end{tabular}

Table 6. Score lesions

\begin{tabular}{cccc}
\hline Score lesions & Group A $(\mathrm{N}=10)$ & Group B $(\mathrm{N}=20)$ & $P$ \\
\hline 28 days old & 3.1 & 2.6 & 0.249 \\
35 days old & 1.8 & 2.2 & 0.205 \\
\hline
\end{tabular}

\section{Discussions}

We compared two different IBD vaccination programs in maternal antibody positive broilers. Their efficacy by means of rapid and uniform IBD antibody immune response and interference to ND vaccination was investigated. Furthermore, the vaccines induced lesion development and effect on the size of the BF were compared.

Maternally derived IBD antibodies at the day of the $1^{\text {st }}$ IBD vaccination in all housing units in farm 2 were checked and they were confirmed not significantly different. That mean both groups had the same starting point as the basis for comparing the increase of IBD antibodies later.

Young chickens are protected by maternal antibodies and then by active immunity which is induced by vaccination. There is a gap of immunity when maternal antibodies decrease to below protective levels and active immunity has not increased to the level of protection (Le Gros et al., 2009). To shorten this gap, a better IBD vaccine will be able to induce antibodies faster (Jackwood \& Sommer, 1999; Van den Berg et al., 2000). Our study showed that the M.B. strain vaccine was able to induce faster and higher IBD antibody titers (Figure 2). If we choose the titer 1500 which is the titer of live IBD vaccines protecting against IBDV infection as the baseline (Bughio et al., 2017), at 28 days old, most of the chickens in group A had a titer above 1500 (72.5\%), while most of the chickens in group B had a titer below 1500 (only $30 \%$ above 1500) (Figure $2 \& 3$ ). At 35 days of age, both groups had a good increase in titer and were protected (group A: 95\%; group B: $85 \%)$.

Another vaccination strategy to control a disease is to use a vaccine to induce a uniform active immune response in all individual of the flock. In this way, the viruses in the field have no chance to attach, replicate and multiply at an extremely large number in any chickens. In addition, they have no chance to infect the other chickens in that flock. Therefore, a superior vaccine will produce better uniformity (CV is lower). The uniformity of both groups was improved from 28 days to 35 days of age. At both stages, the uniformity of group A was better than group B (group A: $48.66 \%$ decreased to $28.51 \%$; group B: $99.47 \%$ decreased to $47.25 \%$ ).

The IBD is characterized by immunosuppression and mortality in chickens of 3 to 6 weeks of age (Eterradossi \& Saif, 2008; Sellaoui et al., 2012; Khenenou et al., 2017). Therefore, one of the common concerns for using live IBD vaccines is the cause of immunosuppression. At this young age, if chickens have immunosuppression, they will not be able to induce an immune response to other antigens such as ND (Allan et al., 1972; Van den Berg et al., 2000). In this study, both groups were using the same ND vaccine program. At 21days of age, group A had a statistically higher ND titers $(P<0.001)$ and more uniformity than group B (Table 3). Hence, the M.B. strain vaccine did not adversely affect immune response ability. It also may increase the health of the chickens, which enabled the chickens to have a better immune response to ND (Figure 4). 


\section{IBD - Elisa idexx}

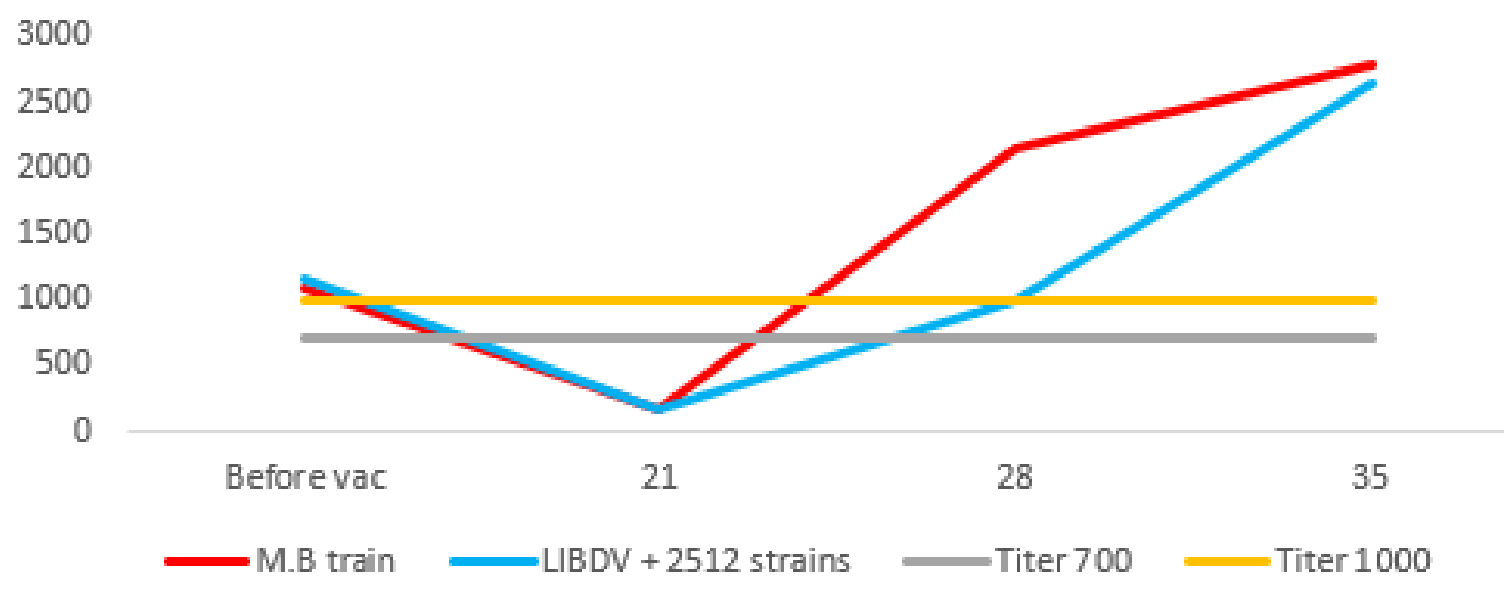

Figure 3. Induction of circulating IBD antibodies.

\section{ND - HI test}

7.00

6.00

5.00

4.00

3.00

2.00

1.00

0.00

\section{1 days old}

\section{8 -days old}

35 days old

GMT-Group A

GMT - Group B

Figure 4. Induction of circulating ND antibodies.

The target organ of IBD viruses is the BF (Khenenou et al., 2017; Farhanah et al., 2018). IBD viruses need to travel through the chicken's body and only when they locate in the BF, will they be able to replicate and infect the chicken (Farhanah et al., 2018). During the gap of immunity, the chickens are at high risk for IBD infection due to the low level of both maternal antibody and humoral immunity (Lazarus et al., 2008; Le Gros et al., 2009). At this stage, a good
IBD vaccine will protect chickens by rapidly locating the vaccine viruses in $\mathrm{BF}$ leaving no space left in the BF for field viruses to locate. Therefore, during the gap of immunity, the speed of location of vaccine viruses in $\mathrm{BF}$ is very important to protect chickens from IBD (Rautenshlein et al., 2005). In other words, a better IBD vaccine has a faster location of its vaccine viruses in the BF. During the development of the chicken, its BF is shrinking over time. It can also shrink due to the 

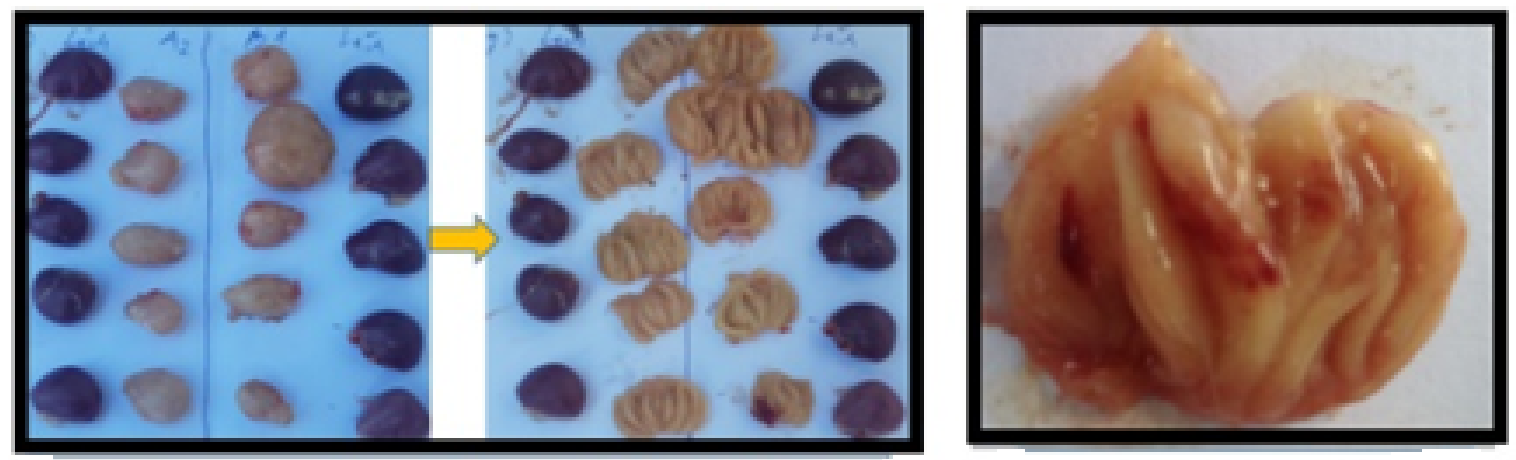

Left $=$ good uniform (group A), right = bad uniform (group B)

Sub-clinical sign (blood spots)

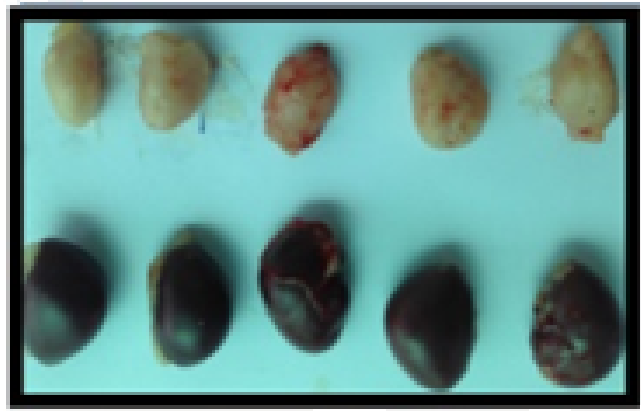

Good uniform, and smaller than spleen group

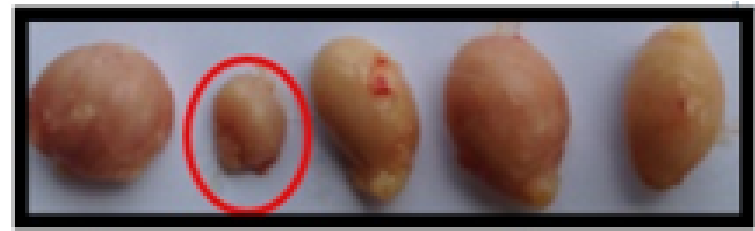

Group B bad uniformity

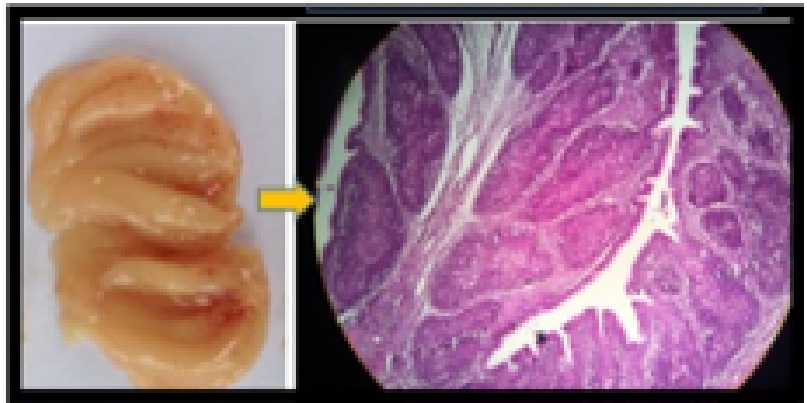

Blood spots (group B, 28 days old, 3 score of lesion)

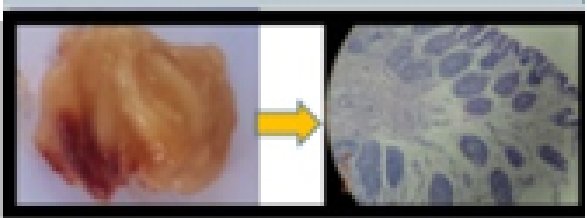

Blood spots (group B, 28 days old, 3 score of lesion)

Figure 5. Sub clinical sign of BF and bursa size vs. spleen size.

location and replication of IBD viruses (Moraes et al., 2004). When the chickens are vaccinated with live IBD vaccine, these vaccine viruses are replicating in the bursa. As a result of this replication, the chicken's immune system is reacting with a humoral antibody response, which will protect the bird from the field strain. One of the signs of this replication is a change in the bursa size the bursa is getting smaller (Moraes et al., 2004; Eterradossi \& Saif, 2008). Therefore, the bursa size of a good IBD vaccine is smaller than normal and it has to be uniform, which means the vaccine provides good titer uniformity leading to uniformity in protection. When the bursa size is not uniform this means the protection is not uniform leading to poor protection and IBD can be sub- clinical or you may have a clinical outbreak. Our study found that at 28 days of age, the BI and CV of group A was smaller than group B, but at 35 days of age, the BI of both groups were similar. This indicated that the LIBDV and 2512 strains located and replicated in the BF later than the M.B. strain. They could only catch up with the M.B. strain at 35 days of age. However, in both stages (28 and 35 days), The M.B. strain was always more uniform than the other strains. Consistently, all data in Table 5 and Figure 5 indicated that the M.B. strain had better uniformity. One more time, it was confirmed that the IDB antibody titers of group A were much more uniform than group B (Figure 2). 


\section{Conclusions}

Comparing different vaccine strains (M.B. strain vs. LIBDV and 2512 strain), the M.B. strain produced better protection for IBD in terms of shortening the immune gap, locating earlier in the $\mathrm{BF}$, inducing higher and more uniform immune responses, and not causing immunosuppression.

\section{References}

Alkie, T. N., \& Rautenschlein, S. (2016). Infectious bursal disease virus in poultry: current status and future prospects. Veterinary Medicine: Research and Reports 7, 9-18.

Allan, W. H., Faragher, J. T., \& Cullen, G. A. (1972). Immunosuppression by the infectious bursal agent in chickens immunized against Newcastle disease. The Veterinary Record 90(18), 511-512.

Allan, W. H., \& Gough, R. E. (1974). A standard haemagglutination inhibition test for Newcastle disease. (1) A comparison of macro and micro methods. Veterinary Record 95(6), 120-123.

Berg, T. P. (2000). Acute infectious bursal disease in poultry: a review. Avian Pathology 29(3), 175-194.

Berg, T. P., \& Meulemans, G. (1991). Acute infectious bursal disease in poultry: protection afforded by maternally derived antibodies and interference with live vaccination. Avian Pathology 20(3), 409-421.

Bughio, E., Jatoi, A. S., Memon, M., Bughio, R., Khoso, P. A., Khoso, Z. A., \& Baloch, A. A. (2017). Effect of age and route of administration on the efficacy of live infectious bursal disease vaccines in broiler. Sarhad Journal of Agriculture 33, 232-239.

De Wit, J . J. (2001). Gumboro disease: estimation of optimal time of vaccination by the Deventer formula. $A n$ nual Report and Proceedings of COST Action 839: Immunosuppressive Viral Diseases in Poultry (170-178). Deventer, the Netherlands: Animal Health Service.

Eterradossi, N., \& Saif, Y. M. (2008). Infectious Bursal Disease. In Swayne, D. E. (Ed.). Disease of poultry (13 ${ }^{\text {th }}$ ed., 219-245). Iowa, USA: John Wiley \& Sons.

Fantay, H., Balcha, E., Tesfay, A., \& Afera, B. (2015). Determining optimum time for administration of live intermediate vaccine of infectious bursal disease to chickens at mekelle farm. Journal of Veterinary Science and Technology 6(3), 223.

Farhanah, M. I., Yasmin, A. R., Khanh, N. P., Yeap, S. K., Hair-Bejo, M., \& Omar, A. R., (2018). Bursal immunopathology responses of specific pathogen free chickens and red jungle fowl infected with very virulent infectious bursal disease virus. Archives of Virology 163(8), 2085-2097.
Fischer, A. H., Jacobson, K. A., Rose, J., \& Zeller, R. (2008). Paraffin embedding tissue samples for sectioning. Cold Spring Harb Protoc. Doi:10.1101/pdb.prot4989.

Gardin, Y., Palya, V., Cazaban, C., Lozano, F., Alva, B., El Attrache, J., \& Dorsey, M. K. (2011). Vaccines and vaccinations against Gumboro disease: the key points. Retrieved September 29, 2018, from https://en.engormix.com/poultryindustry/articles34914.htm.

Gomes, L., Ashash, U., \& Banet-Noach, C. (2015). A field study on broiler flocks in Brazil to evaluate zootechnical parameters, molecular epidemiology, and condemnation index with the use of Live IBD Vaccine versus HVT-IBD Vector Vaccine. Retrieved September 29, 2018, from http://www.wvpa.net/pdfs/articles/WVPC-2015AB058.pdf.

Jackwood, D. (2017). Optimizing immunity in 'no antibiotics ever' and 'reduced use' broiler flocks. Retrived September 29, 2018, from https://poultryhealthtoday.com/opt.pdf

Jackwood, D. J., \& Sommer, S. E. (1999). Restriction fragment length polymorphisms in the VP2 gene of infectious bursal disease viruses from outside the United States. Avian Diseases 43(2), 310-314.

Khenenou, T., Bougherara, M., Melizi, M., \& Lamraoui, R. (2017). Histomorphological Study of the Bursae of Fabricius of Broiler Chickens during Gumboro Disease in Algeria Area. Global Veterinaria 18(2), 132-136.

Lazarus, D., Pasmanik-Chor, M., Gutter, B., Gallili, G., Barbakov, M., Krispel, S., \& Pitcovski, J. (2008). Attenuation of very virulent infectious bursal disease virus and comparison of full sequences of virulent and attenuated strains. Avian Pathology 37(2), 151-159.

Le Gros, F. X., Dancer, A., Giacomini, C., Pizzoni, L., Bublot, M., Graziani, M., \& Prandini, F. (2009). Field efficacy trial of a novel HVT-IBD vector vaccine for 1-day-old broilers. Vaccine 27(4), 592-596.

Moraes, H., Salle, C., Padilha, A., Nascimento, V., Souza, G., Pereira, R., Artencio, J., \& Salle, F. (2004). Infectious bursal disease: evaluation of pathogenicity of commercial vaccines from Brazil in specific pathogen free chickens. Brazilian Journal of Poultry Science 6(4), 243-247.

Muller, H., Mundt, E., Eterradossi, N., \& Islam, M. R. (2012). Current status of vaccines against infectious bursal disease. Avian pathology 41(2), 133-139.

Muskett, J., Hopkins, I., Edwards, K., \& Thornton, D. (1979). Comparison of two infectious bursal disease vaccine strains: efficacy and potential hazards in susceptible and maternally immune birds. The Veterinary Record 104(15), 332-334. 
Rautenschlein, S., Kraemer, C. H., Vanmarke, J., \& Montiel, E. (2005). Protective efficacy of intermediate and intermediate plus infectious bursal disease virus (IBDV) vaccines against very virulent IBDV in commercial broilers. Avian Disseases 49(2), 231-237.

Sellaoui, S., Alloui, N., Mehenaoui, S., \& Djaaba, S. (2012). Evaluation of size and lesion scores of bursa cloacae in broiler flocks in Algeria. Journal of World's Poultry Research 2(3), 37-39.
Van den Berg, T. P., Eterradossi, N., Toquin, D., \& Meulemans, G. (2000). Infectious bursal disease (Gumboro disease). Revue scientifique et technique 19(2), 509-543. 